\title{
Solid solutions vs. solid dispersions: the impact of formulation parameters
}

\author{
Soluções sólidas vs. dispersões sólidas: impacto dos parâmetros de formulação \\ Joana Mota ${ }^{1,2}$ \\ ${ }^{1} \mathrm{CBiOS}$ - Research Center for Biosciences and Health Technologies, \\ U Lusófona, Campo Grande 376, 1749-024 Lisboa, Portugal \\ ${ }^{2}$ College of Pharmacy, Freie Universität Berlin, Berlin, Germany \\ E-mail: joana.mota@ulusofona.pt
}

\begin{abstract}
Matrix films were prepared with drugs with different aqueous solubility (metoprolol tartrate, ibuprofen and diclofenac $\mathrm{Na}$ ) and different polymers (ethylcellulose, Eudragit RS and Eudragit RL). Solid solutions (drug dissolved) and solid dispersions (drug dispersed) were obtained. The drug release was studied as a function of drug type and loading, additive type and amount and polymer type. Drug release from the matrices was not in accordance with drug aqueous solubility, but rather with the physical state of the drug in the matrix. Increasing the drug loading increased the drug release rate in a monotonical fashion for the solid solution. In contrast, increasing the drug loading with the solid dispersion had almost no effect until $30 \%$. An increase in speed was only registered after the drug release significantly increased. The addition of hydrophilic additives mainly increased the initial phase of the release profile, with no effect on the plateau. On the contrary, polyethylene glycol 1500 was shown to decrease the release of diclofenac $\mathrm{Na}$ due to entrapment of the drug in the hydrophilic additive domains. The effect of polymer type on ibuprofen release correlated well with the partition of the drug into the polymer. In the case of metoprolol tartrate, drug release was much faster from Eudragit RS than EC matrices due to the formation of an amorphous mixture with Eudragit RS polymer.
\end{abstract}

Keywords: solid solution, solid dispersion, cast matrix films, controlled release

\section{Resumo}

Filmes matriciais foram preparados com fármacos com diferentes solubilidades aquosas (tartarato de metoprolol, ibuprofeno e diclofenac Na) e diferentes polímeros (etilcelulose, Eudragit RS e Eudragit RL). Foram obtidas soluções sólidas (fármaco dissolvido) e dispersões sólidas (fármaco disperso). A libertação dos fármacos foi estudada em função do tipo de fármaco, doseamento, tipo e quantidade de aditivo e do tipo de polímero. A libertação dos fármacos não foi de acordo com a sua solubilidade aquosa, mas sim com o estado físico do fármaco na matriz. $\mathrm{O}$ incremento do conteúdo do fármaco na matriz aumentou a velocidade de libertação monotonicamente para a solução sólida. Em contraste, com a dispersão sólida, o aumento da dosagem de fármaco não originou quase nenhum efeito até $30 \%$, registando-se apenas, posteriormente, um acréscimo na sua velocidade. A inclusão de aditivos hidrofílicos aumentou principalmente a fase inicial do perfil de libertação do fármaco e não produziu efeito no plateau. Por outro lado, polietilenoglicol 1500 diminuiu a libertação de diclofenac Na devido ao aprisionamento do fármaco nos seus domínios. O efeito do tipo de polímero na libertação de ibuprofeno esteve relacionado com a partição do fármaco com o polímero. No caso do tartarato de metoprolol, a libertação foi muito mais rápida do da matriz de Eudragit RS do que de EC, devido à formação de uma mistura amorfa.

Palavras-chave: solução sólida, dispersão sólida, filmes matriciais, libertação controlada. 


\section{Introduction}

Coated multiparticulates may be formulated as matrix and reservoir systems. In coated matrix systems, the pellets are coated with a drug and polymer solution whereas in reservoir systems the pellets are first coated with a drug layer and after with a polymer solution. Matrix coated systems present several advantages: they are easy to manufacture and low in cost (1 step process), there is a lower risk of dose dumping (if the coating accidentally ruptures) and there is the possibility of improving the aqueous drug solubility. Furthermore, drugpolymer interactions can occur and these bring benefits in terms of mechanical properties such the plasticizing effect. ${ }^{[1,2]}$ The main disadvantages include fast initial release ${ }^{[3]}$ and incomplete release in a defined time.

In a matrix system the drug and polymer are dissolved or dispersed in a common solvent and upon solvent evaporation, a solid solution (drug dissolved in the polymer) or a solid dispersion (drug dispersed in the polymer) or a combination of both is obtained.

The objective of this study is to identify the key parameters affecting drug release from solid solutions and solid dispersions in cast films, in order to predict the release from matrix-coated pellets.

\section{Material and Methods}

\section{Materials}

Metoprolol tartrate (Moehs, Barcelona, Spain), diclofenac sodium (Farchemia, Treviglio, Italy) and ibuprofen (BASF AG, Germany), hydroxypropylmethylcellulose (HPMC) (Methocel E5, Colorcon, England), hydroxypropylcellulose (HPC) (Klucel EXF and JF, Hercules GmbH, Germany), povidone K90 (BASF AG, Germany), polyethylene glycol (PEG) 1500 (Lutrol E, Germany), ethylcellulose (Ethocel Standard premium 10, DOW Chemical Company, USA), ammonio methacrylate copolymer type A (Eudragit RS) and ammonio methacrylate copolymer type B (Eudragit RS). All other chemicals were of reagent analytical grade.

\section{Preparation of cast matrix films}

Matrix films were prepared by dissolving diclofenac $\mathrm{Na}(30 \mathrm{cg} / \mathrm{g})$, ibuprofen (between $10 \mathrm{cg} / \mathrm{g}$ and $50 \mathrm{cg} / \mathrm{g}$ ) and metoprolol tartrate (between $10 \mathrm{cg}$ and $50 \mathrm{cg} / \mathrm{g}$ ) in isopropanol: water, in the respective proportion $88 \mathrm{~g}$ : $12 \mathrm{~g}$. After the addition of the polymer (ethylcellulose, $6 \mathrm{cg} / \mathrm{g}$; Eudragit RS and Eudragit RL, $10 \mathrm{cg} / \mathrm{g}$ ), HPC

\section{Introdução}

Sistemas multiparticulados revestidos podem ser formulados como sistemas matriciais ou reservatórios. Nos sistemas com revestimento matricial, os pellets são revestidos por uma solução de fármaco e polímero, enquanto nos sistemas reservatório os pellets são revestidos, primeiramente, por uma camada de fármaco e, posteriormente, por uma camada de polímero. Os primeiros apresentam diversas vantagens, como fácil fabrico e baixo custo (1 passo no processo), menor risco de libertação descontrolada (ruptura da membrana) e a possibilidade de aumento da solubilidade aquosa do fármaco. Além disso, interacções fármaco-polímero podem ocorrer e trazer benefícios em termos de propriedades mecânicas, entre as quais se destaca o efeito plasticizante ${ }^{[1,2]}$ As principais desvantagens incluem a libertação inicial rápida ${ }^{[3]}$ e a libertação incompleta.

Num sistema matricial, o fármaco e o polímero são dissolvidos ou dispersos num solvente comum e, após evaporação do solvente, uma solução sólida (fármaco dissolvido no polímero) ou uma dispersão sólida (fármaco disperso no polímero) - ou, então, uma combinação de ambos - é obtida.

O objetivo deste estudo é identificar os principais parâmetros que afectam a libertação do fármaco a partir de soluções sólidas e dispersões sólidas em filmes (membranas matriciais), para poder prever a libertação de pellets revestidos por matrizes.

\section{Materiais e Métodos}

\section{Materiais}

Tartarato de metoprolol (Moehs, Espanha), diclofenac $\mathrm{Na}$ (Farchemia, Itália) e ibuprofeno (BASF AG, Alemanha), hidroxipropilmetilcelulose (HPMC) (Methocel E5, Colorcon, Reino Unido), hidroxipropilcelulose (HPC) (Klucel EXF e JF, Hercules GmbH, Alemanha), polivinilpirrolidona (BASF AG, Alemanha), polietilenoglicol (PEG) 1500 (Lutrol E, Alemanha) etilcelulose (Ethocel Standard premium 10, DOW Chemical Company, EUA), copolímero de metacrilato de amónio tipo $A$ (Eudragit RL) e tipo B (Eudragit RS). Os restantes reagentes foram de grau analítico

\section{Preparação de filmes matriciais}

Os filmes matriciais foram preparados por dissolução do diclofenac Na $(30 \mathrm{cg} / \mathrm{g})$, ibuprofeno (entre $10 \mathrm{cg} / \mathrm{g}$ e $50 \mathrm{cg} / \mathrm{g}$ ) e tartarato de metoprolol (entre $10 \mathrm{cg} / \mathrm{g}$ e $50 \mathrm{cg} / \mathrm{g}$ ) em álcool isopropílico: água, na respectiva proporção 88 g: 12 g). Depois da adição e dissolução dos polímeros (etilcelulose, $6 \mathrm{cg} / \mathrm{g}$; Eudragit RS e Eudragit RL, $10 \mathrm{cg} / \mathrm{g}$ ), HPC JF, PVP K90, manitol entre 7 
JF, PVP K90, manitol, between $7 \mathrm{cg} /$ and $28 \mathrm{cg} / \mathrm{g}$ and PEG 1500, between $6 \mathrm{cg} / \mathrm{g}$ and $30 \mathrm{cg} / \mathrm{g}$ were optionally added. After overnight stirring, matrix solutions/dispersions were cast on petri dishes, covered with paper and dried at room temperature for 2 days. The thickness of the films was measured using thickness gauge (Minitest 600, Erichsen, Hemer, Germany).

\section{Drug release from matrix films}

The edges of the films were sealed with vaseline to avoid drug diffusion through the film ends. Drug release was conducted in $900 \mathrm{ml}$ (diclofenac $\mathrm{Na}$ and ibuprofen) and $500 \mathrm{ml}$ (metoprolol tartrate) phosphate buffer pH 6,8 $\left(75 \mathrm{rpm}, 37^{\circ} \mathrm{C}, n=3\right)$ in a horizontal shaker (GFL 3033). At predetermined time intervals, $3 \mathrm{ml} \mathrm{sam}$ ples were withdrawn and analyzed (directly or after appropriate dilution) with Shimadzu UV-2101PC UVVis Scanning spectrophotometer (Shimadzu Europe, Germany) giving the following results: diclofenac $\mathrm{Na}$, $\lambda=275 \mathrm{~nm}$; ibuprofen, $\lambda=264 \mathrm{~nm}$; metoprolol tartrate, $\lambda=274 \mathrm{~nm}$. The osmolality was determined based on the freezing point of the solutions compared to pure water.

\section{Solubility measurements}

Excess amounts of diclofenac $\mathrm{Na}$ and ibuprofen were placed in contact with phosphate buffer $\mathrm{pH} 6,8$ in a horizontal shaker (GFL 3033) (75 rpm, $37{ }^{\circ} \mathrm{C}, n=3$ ) for at least $72 \mathrm{~h}$. Every $24 \mathrm{~h}$, samples were withdrawn, filtered and the $\mathrm{pH}$ of the saturated solution was adjusted with sodium hydroxide solution. The samples were then analyzed for the drug content after equilibrium was reached.

\section{Determination of the drug content}

The residual drug content in the films was determined after extraction in ethanol (96\%) for selected samples (diclofenac Na, $\lambda=282 \mathrm{~nm}$; ibuprofen, $\lambda=264 \mathrm{~nm}$; metoprolol tartrate, $\lambda=276 \mathrm{~nm}$ ).

\section{Drug partition into polymer}

Ibuprofen partition into ethylcellulose, Eudragit RS and Eudragit RL was determined as follows: a known amount of the drug was dissolved in a known amount of phosphate buffer $\mathrm{pH} 6,8$. Accurately weighed polymer was added to the drug solution and stirred over a period of $24 \mathrm{~h}\left(75 \mathrm{rpm}, 37^{\circ} \mathrm{C}, n=3\right)$ in a horizontal shaker (GFL 3033). $3 \mathrm{ml}$ samples were withdrawn and analyzed (directly or after appropriate dilution) with UV spectrophotometry (Shimadzu UV-2101PC UV-Vis Scanning spectrophotometer; Shimadzu Europe, Germany).

Drug partition into the polymer was calculated as follows:

where $\mathrm{C}_{\mathrm{i}}$ is the initial drug concentration and $\mathrm{C}_{\mathrm{f}}$ is the final drug concentration. $\mathrm{cg} /$ e $28 \mathrm{cg} / \mathrm{g}$ e PEG 1500 entre $6 \mathrm{cg} / \mathrm{g}$ e $30 \mathrm{cg} / \mathrm{gforam}$ opcionalmente adicionados. Após agitação durante a noite, as soluções e dispersões foram colocadas em caixas de petri, cobertas com papel secas à temperatura ambiente ( 2 dias). A espessura dos filmes foi medida (Minitest 600, Erichsen, Hemer, Alemanha).

\section{Ensaio de libertação in vitro}

Os bordos dos filmes foram selados com vaselina para evitar difusão dos fármacos através da orla dos filmes. $\mathrm{O}$ ensaio foi realizado com $900 \mathrm{ml}$ (diclofenac $\mathrm{Na}$ e ibuprofeno) e $500 \mathrm{ml}$ (tartarato de metoprolol) em tampão fosfato $\mathrm{pH} 6,8\left(75 \mathrm{rpm}, 37^{\circ} \mathrm{C}, n=3\right)$, numa incubadora com agitação horizontal (GFL 3033). Em intervalos de tempo pré-determinados, foram retiradas amostras de $3 \mathrm{ml} \mathrm{e}$ analisadas (directamente ou após diluição apropriada) num espectrofotómetro UV-visível (Shimadzu UV-2101PC UV-Vis Scanning spectrophotometer; Shimadzu Europe, Alemanha). Diclofenac Na, $\lambda=275 \mathrm{~nm}$; ibuprofeno, $\lambda=264 \mathrm{~nm}$ e tartarato de metoprolol, $\lambda=274 \mathrm{~nm}$. A osmolalidade foi determinada através da medida do ponto de congelamento da solução em comparação com a água.

\section{Determinação da solubilidade}

Uma quantidade em excesso de diclofenac $\mathrm{Na}$ e ibuprofeno foi colocada em tampão fosfato $\mathrm{pH}$ 6,8 (75 rpm, $37^{\circ} \mathrm{C}, n=3$ ) numa incubadora com agitação horizontal (GFL 3033) pelo menos durante $72 \mathrm{~h}$. A cada $24 \mathrm{~h}$ foram recolhidas amostras, filtradas e o $\mathrm{pH}$ das soluções saturadas foi ajustado com uma solução de hidróxido de sódio. As amostras foram analisadas directamente ou, após diluição, num espectrofotómetro UV-visível (Shimadzu UV-2101PC UV-Vis Scanning spectrophotometer; Shimadzu Europe, Alemanha).

\section{Determinação do conteúdo de fármaco}

A quantidade residual de fármaco nos filmes foi determinada após extracção em etanol (96 \%) com amostras selecionadas (diclofenac Na, $\lambda=282 \mathrm{~nm}$; ibuprofeno, $\lambda=264 \mathrm{~nm}$; tartarato de metoprolol, $\lambda=276 \mathrm{~nm}$ ).

\section{Coeficiente de partição entre o fármaco e polímero}

A partição do fármaco para etilcelulose, Eudragit RS e Eudragit RL foi determinada como se segue: uma quantidade conhecida de fármaco foi dissolvida numa quantidade conhecida de tampão fosfato $\mathrm{pH}$ 6,8. Uma quantidade rigorosamente pesada de polímero foi adicionada à solução anterior e deixada em agitação durante $24 \mathrm{~h}\left(75 \mathrm{rpm}, 37^{\circ} \mathrm{C}, n=3\right)$ numa incubadora horizontal (GFL 3033). Amostras de $3 \mathrm{ml}$ foram retiradas e analisadas (directamente ou após diluição apropriada) num espectrofotómetro UV-visível (Shimadzu UV-2101PC UV-Vis Scanning spectrophotometer; Shimadzu Europe, Alemanha).

O coeficiente de partição do fármaco para o polímero foi determinado:

$\mathrm{C}_{\mathrm{i}}$ corresponde à concentração inicial de fármaco e $\mathrm{C}_{\mathrm{f}}$ corresponde à concentração final 
Differential scanning calorimetry (DSC)

Thermograms of PEG 1500, ethylcellulose, diclofenac $\mathrm{Na}$, matrices of ethylcellulose: diclofenac $\mathrm{Na}$ 60:40 (cast film and physical mixture) with and without PEG 1500 , were obtained by differential scanning calorimetry (Mettler DSC 821) and STAR software (Mettler Toledo, Giessen, Germany) to determine the melting point. The samples (between $10 \mathrm{mg}$ and $20 \mathrm{mg}$ ) were sealed in aluminum pans. All tests were run under a nitrogen atmosphere at a scanning rate of $5^{\circ} \mathrm{C} / \mathrm{min}$ over a temperature range of $0{ }^{\circ} \mathrm{C}$ to $80^{\circ} \mathrm{C}$.

\section{Microscopic analysis}

Matrix films were analyzed by polarizing light microscopy. The microscope was equipped with an imaging system (EasyMeasure; INTEQ Informationstechnik, Germany).

\section{Results and Discussion}

\section{Effect of drug type}

Drug release from ethylcellulose (EC) matrix films was in the following order: diclofenac $\mathrm{Na}>$ ibuprofen $>$ metoprolol tartrate (Fig. 1). Interestingly, the ranking order of the observed release rates did not follow the ranking order of aqueous solubility (metoprolol tartrate, 3630 $\mathrm{mg} / \mathrm{ml}^{[1]}{ }^{[1]}>$ ibuprofen, $11,1 \mathrm{mg} / \mathrm{ml}>$ diclofenac $\mathrm{Na}, 1.0$ $\mathrm{mg} / \mathrm{ml}$ ). In addition, drug solubility in the matrix was as follows: ibuprofen, $50 \mathrm{cg} / \mathrm{g}>$ diclofenac $\mathrm{Na}, 10 \mathrm{cg} / \mathrm{g}>$ metoproplol tartrate, $5 \mathrm{cg} / \mathrm{g}$ (determined by the occur-

\section{Calorimetria diferencial de varrimento}

Termogramas de PEG 1500, etilcelulose, diclofenac $\mathrm{Na}$, matrizes de etilcelulose:diclofenac $\mathrm{Na}$ 60:40 (filme e mistura física) com e sem PEG 1500 foram obtidos por calorimetria diferencial de varrimento (Mettler DSC 821) e STAR software (Mettler Toledo, Alemanha). Amostras (entre $10 \mathrm{mg}$ e $20 \mathrm{mg}$ ) foram seladas em pequenos recipientes de alumínio. Todos os testes foram conduzidos sob atmosfera de nitrogénio a uma velocidade de varrimento de $5^{\circ} \mathrm{C} / \mathrm{min}$ e num intervalo de temperaturas de $0{ }^{\circ} \mathrm{C}$ a $80{ }^{\circ} \mathrm{C}$.

\section{Análise microscópica}

Os filmes foram analisados por microscopia de luz polarizada (EasyMeasure; INTEQ Informationstechnik, Alemanha).

\section{Resultados e Discussão}

\section{Efeito do tipo de fármaco}

A libertação dos fármacos da matriz de etilcelulose foi na seguinte ordem: diclofenac $\mathrm{Na}>$ ibuprofeno $>$ tartarato de metoprolol (Fig. 1). Curiosamente, esta ordem não está de acordo com a ordem da solubilidade aquosa (tartarato de metoprolol, $3630 \mathrm{mg} / \mathrm{ml}^{[1]}>>$ ibuprofeno, $11,1 \mathrm{mg} / \mathrm{ml}>$ diclofenac $\mathrm{Na}, 1.0 \mathrm{mg} / \mathrm{ml}$ ). Além disso, a solubilidade do fármaco na matriz foi: ibuprofeno, 50 $\mathrm{cg} / \mathrm{g}>$ diclofenac Na, $10 \mathrm{cg} / \mathrm{g}>$ metoproplol tartrate, $5 \mathrm{cg} / \mathrm{g}$ (determinado pela ocorrência de cristais de fár-

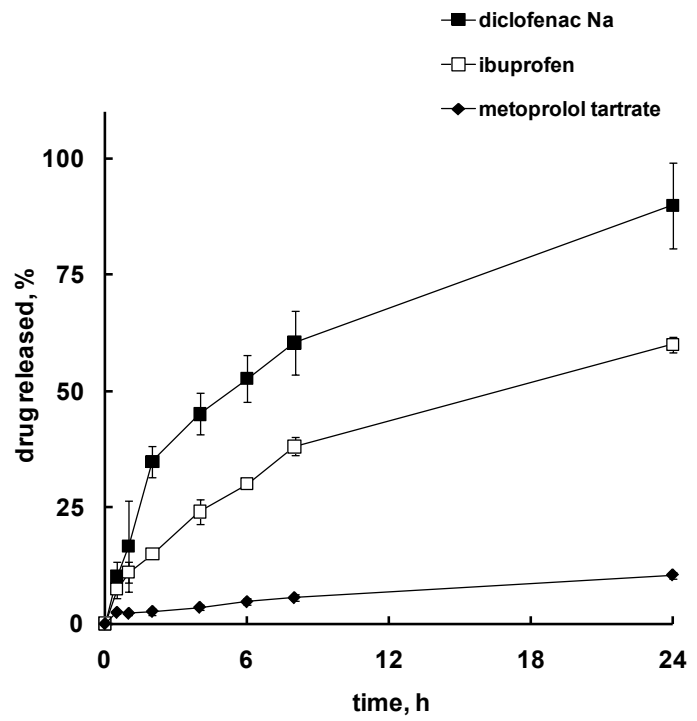

Figure 1 - Effect of drug type on release from ethylcellulose matrix systems (30 cg/g drug loading)

Figura 1 - Efeito do tipo de fármaco na libertação a partir de sistemas matriciais de etilcelulose ( $30 \mathrm{cg} / \mathrm{g}$ de dosagem) 
rence of drug crystals in the cast film). As an example, at $30 \%$ drug loading, diclofenac $\mathrm{Na}$ was dissolved and dispersed (large crystals on matrix surface), ibuprofen was completely dissolved (absence of any crystal) and metoprolol tartrate was mainly dispersed in the matrix (small crystals) (Fig. 2). The fast release of diclofenac $\mathrm{Na}$ was attributed to rapid dissolution and release of drug crystals. Due to the high solubility of ibuprofen in the matrix, diffusion through the matrix was the mechanism controlling drug release. Metoprolol tartrate, although a very high soluble drug, was released very slowly due to drug entrapment in the matrix. maco na matriz). Por exemplo, para uma dosagem de $30 \%$, o diclofenac de $\mathrm{Na}$ está dissolvido e disperso (grandes cristais na superfície da matriz), o ibuprofeno está completamente dissolvido (ausência de cristais) e tartarato de metoprolol está essencialmente disperso na matriz (Fig. 2). A libertação rápida de diclofenac $\mathrm{Na}$ foi resultado de uma rápida dissolução e libertação dos cristais de fármaco. Devido à elevada solubilidade do ibuprofeno no polímero, a difusão através da matriz foi o mecanismo de controlo da libertação. No caso do tartarato de metoprolol, a libertação foi muito lenta devido ao seu aprisionamento na matriz, muito embora o fármaco apresente uma elevada solubilidade aquosa.

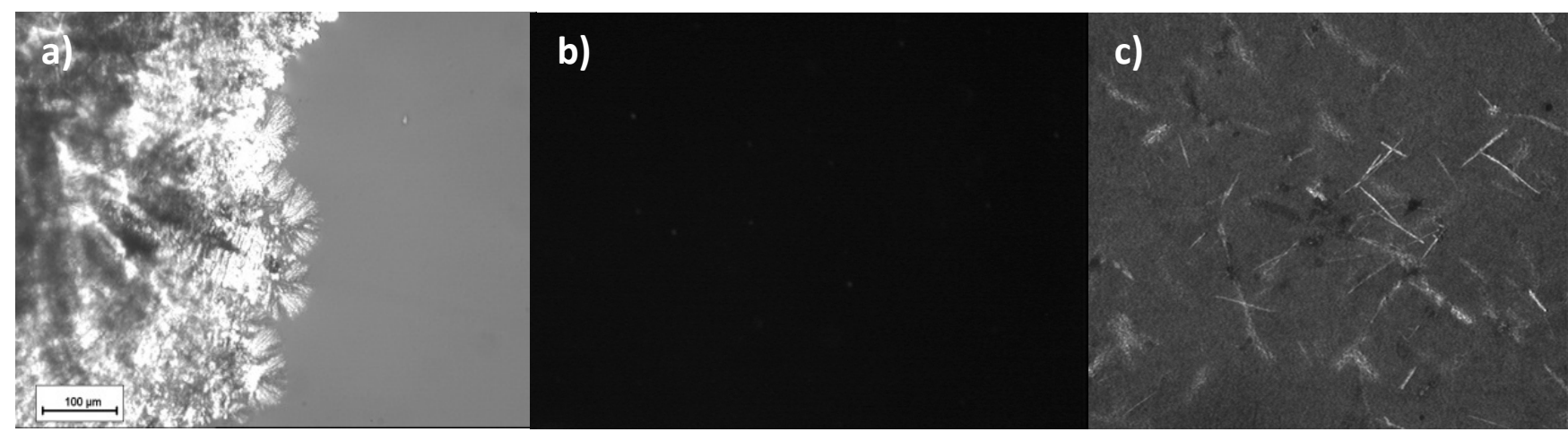

Figure 2 - Polarizing light microscope images from ethylcellulose matrix cast films (30\% drug loading). a) diclofenac $\mathrm{Na}, \mathrm{b}$ ) ibuprofen and c) metoprolol tartrate

Figura 2 - Imagens de microscopia de luz polarizada de filmes matriciais de etilcelulose (30\% de dosagem de fármaco). a) diclofenac $\mathrm{Na}, \mathrm{b}$ ) ibuprofeno e c) tartarato de metoprolol

\section{Effect of drug loading}

\section{$\underline{\text { Solid solution }}$}

Drug release increased monotonically with increased drug loading due to the higher amount of drug able to diffuse in the matrix (Fig 3a). Ibuprofen release could be linearly described by a square root of time relationship, indicating a diffusion-controlled mechanism (Fig. $3 \mathrm{~b})$. Once the drug was dissolved (solid solution), drug diffusion occurred through the polymer for all drug loadings and a constant drug release rate (determined by the slope of the linear portion of the curve of cumulative amount of drug release, versus square root of time) increased in direct proportion to the drug concentration, except with the lowest drug loading (Fig. 3c). The amount of ibuprofen remaining in the matrix at $24 \mathrm{~h}$ was plotted against the initial drug loading and a linear relationship was also obtained. The amount of the drug not released was directly correlated with initial loading. By extrapolation of the curve, approximately $65 \mathrm{cg} / \mathrm{g}$ ibuprofen loading is necessary to achieve complete release (Fig. 3d). On the other hand, it is clear from Fig.

\section{Efeito da dosagem}

\section{Solução sólida}

A libertação do ibuprofeno aumentou monotonicamente com o incremento da dosagem, devido à maior quantidade de fármaco capaz de difundir (Fig. 3a). A libertação do ibuprofeno pode ser linearmente descrita em função da raiz quadrada do tempo, o que indica um mecanismo controlado por difusão. Uma vez que o fármaco está dissolvido, a difusão pura através do polímero ocorre para todas as dosagens e a velocidade de libertação do fármaco (determinada pelo declive da porção linear da curva da quantidade cumulativa de libertação do fármaco versus a raiz quadrada do tempo) aumenta numa proporção directa com a concentração do fármaco, excepto para a menor dosagem (Fig. 3c). A quantidade de ibuprofeno restante na matriz às $24 \mathrm{~h}$ foi representada graficamente em relação à dosagem inicial e uma relação linear também foi obtida. A quantidade de fármaco não libertado é directamente proporcional à dosagem inicial. Por extrapolação da curva, era necessário, aproximadamente, uma dosagem de $65 \mathrm{cg} / \mathrm{g}$ 
3a that increasing the drug loading to $65 \mathrm{cg} / \mathrm{g}$ would also increase the initial drug release.

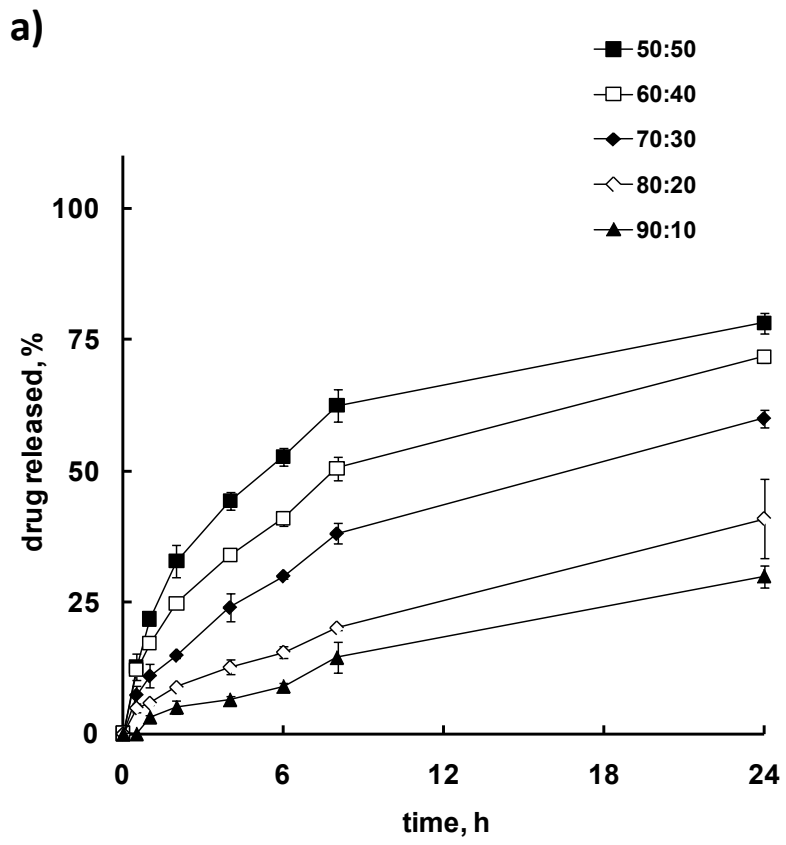

c)

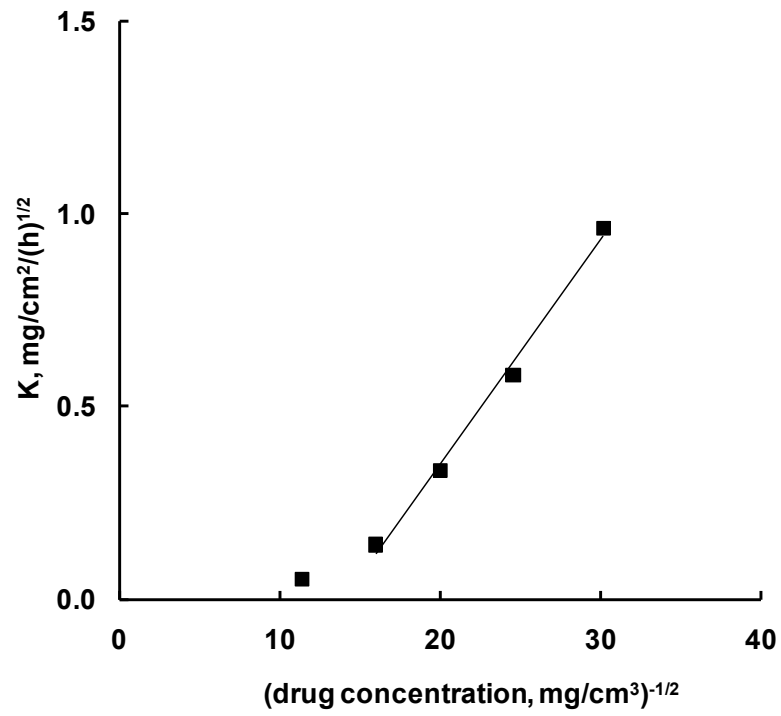

de ibuprofeno para alcançar uma libertação completa (Fig. 3d). Por outro lado, é evidente, a partir da Fig. 3a, que o aumento da dosagem para $65 \mathrm{cg} / \mathrm{g}$ iria aumentar a velocidade inicial de fármaco.

b)

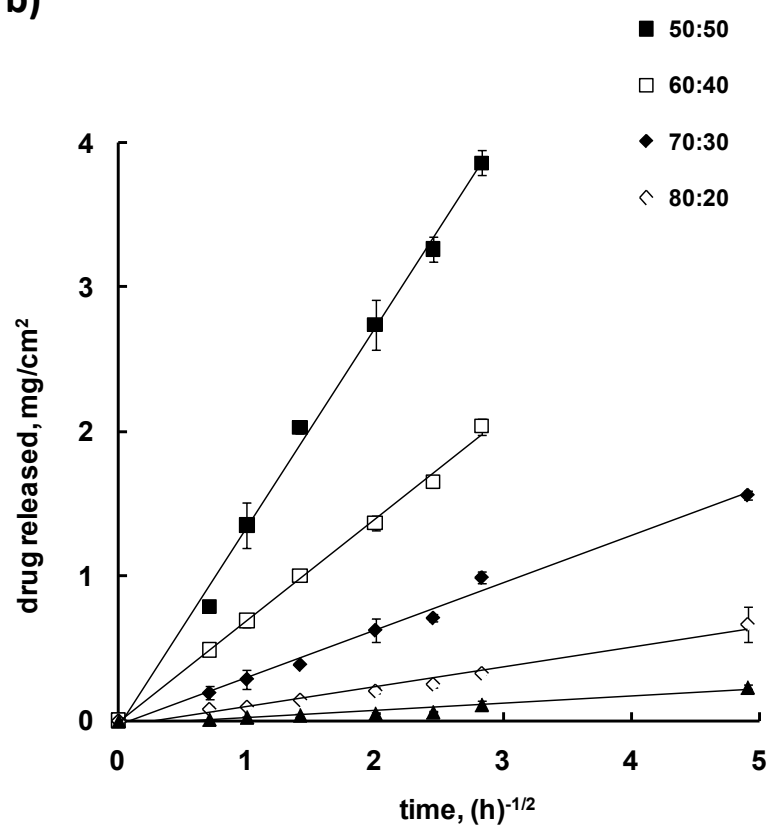

d)

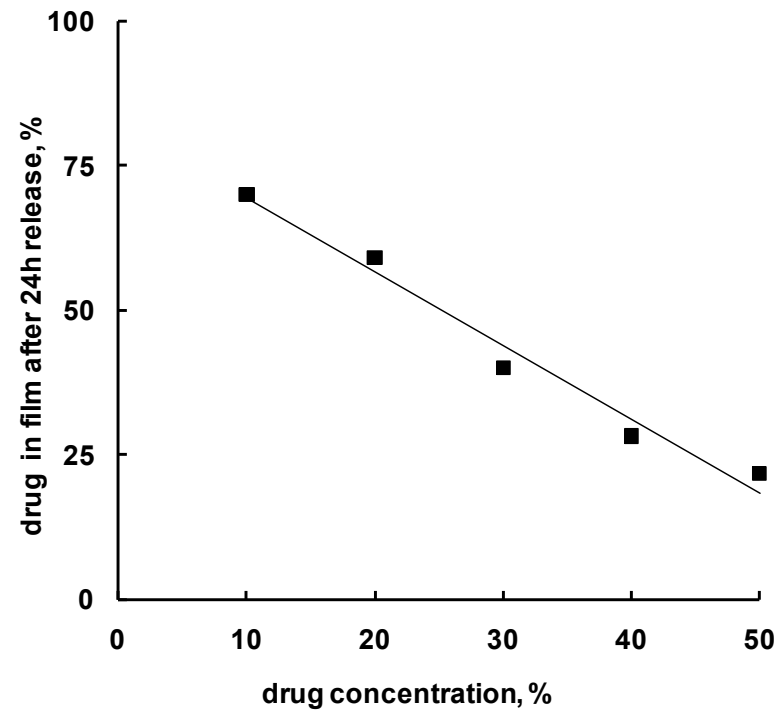

Figure 3 - Effect of ibuprofen loading on ethylcellulose matrix cast films. a) drug release, $\%, b$ ) drug release, $\mathrm{mg} / \mathrm{cm}^{2}$ vs. $\left.(\mathrm{t})^{-1 / 2}, \mathrm{c}\right)$ release rate constant,$(K) \mathrm{mg} / \mathrm{cm}^{2} /(\mathrm{h})^{-1 / 2}$ and d) drug remaining in the matrix at $24 \mathrm{~h}, \%$.

Figura 3 - Efeito do aumento da dosagem de ibuprofeno em filmes matriciais de etilcelulose. a) libertação do fármaco,\%, b) libertação do fármaco, $\left.\mathrm{mg} / \mathrm{cm}^{2} \mathrm{vs} .(\mathrm{t})^{-1 / 2}, \mathrm{c}\right)$ constante de libertação $\left.(K), \mathrm{mg} / \mathrm{cm}^{2} /(\mathrm{h})^{-1 / 2} \mathrm{e} \mathrm{d}\right)$ fármaco restante na matriz após $24 \mathrm{~h}, \%$ 


\section{$\underline{\text { Solid dispersion }}$}

Metoprolol tartrate release was very low up to $30 \%$ of drug loading and then increased with increasing drug loading. However, complete release was not reached (Fig. 4a). Drug release could be described by a square

\section{Dispersão sólida}

A libertação de tartarato de metoprolol foi muito baixa até $30 \%$ de dosagem inicial e posteriormente aumentou com o incremento de dosagem de fármaco. No entanto, a libertação completa não foi alcançada (Fig. 4a). A lib-
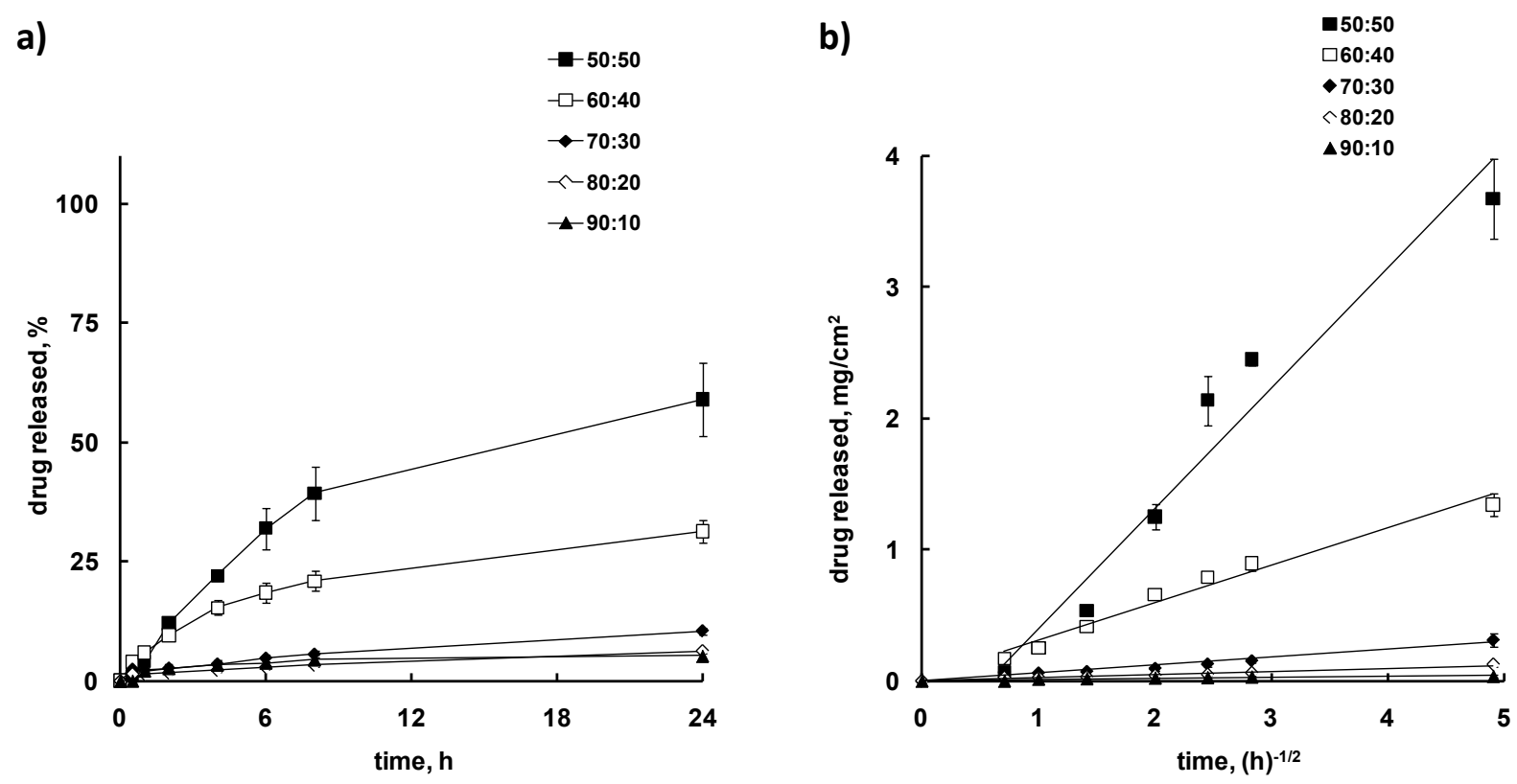

c)

d)
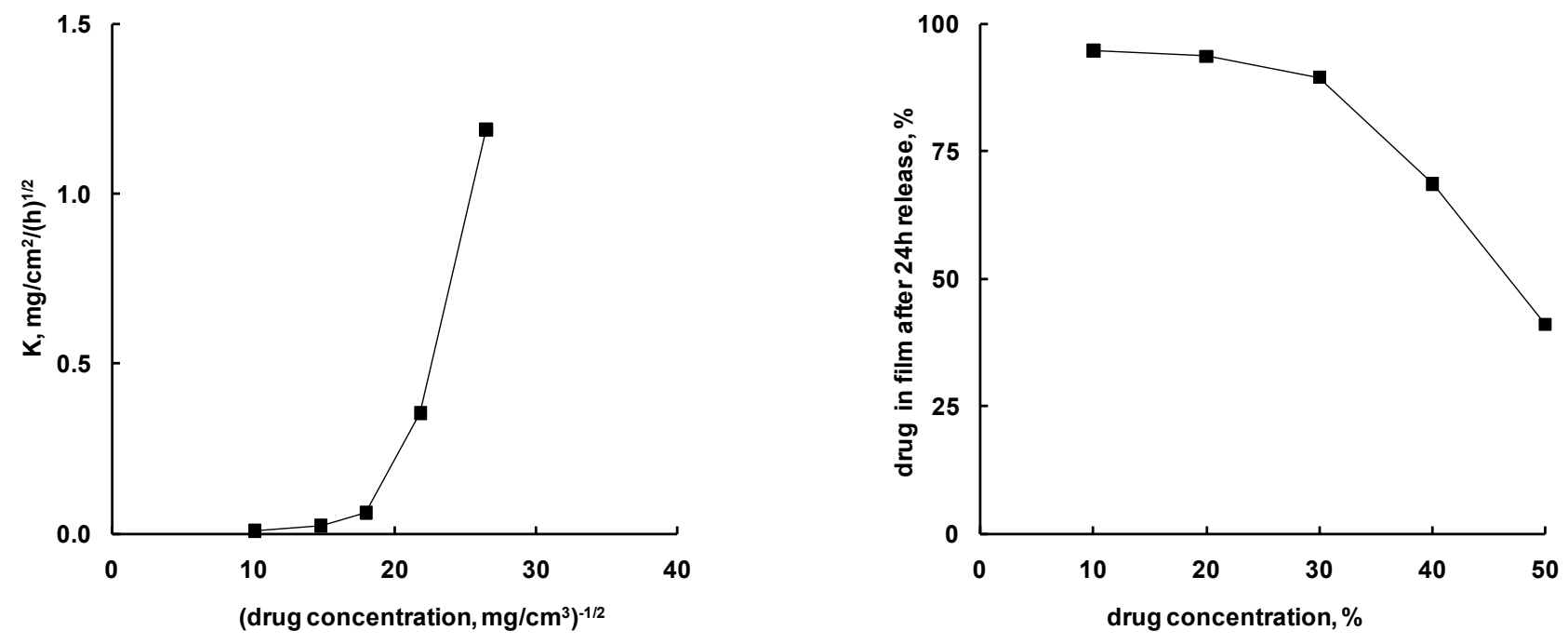

Figure 4 - Effect of metoprolol tartrate loading on ethylcellulose matrix cast films. a) drug release, \%, b) drug release, mg/ $\mathrm{cm}^{2}$ vs. $\left.(\mathrm{t})^{-1 / 2}, \mathrm{c}\right)$ release rate constant $(\mathrm{K}), \mathrm{mg} / \mathrm{cm}^{2} /(\mathrm{h})^{-1 / 2}$ and d) drug remaining in the matrix at $24 \mathrm{~h}, \%$.

Figura 4 - Efeito do aumento da dosagem de tartarato de metoprolol em filmes matriciais de etilcelulose. a) libertação do fármaco, $\%$, b) libertação do fármaco, $\mathrm{mg} / \mathrm{cm}^{2} \mathrm{vs}$. $\left.(\mathrm{t})^{-1 / 2}, \mathrm{c}\right)$ constante de libertação $\left.(\mathrm{K}), \mathrm{mg} / \mathrm{cm}^{2} /(\mathrm{h})^{-1 / 2} \mathrm{e} \mathrm{d}\right)$ fármaco restante na matriz após $24 \mathrm{~h}, \%$. 
root of time relationship, indicating a diffusion controlled mechanism (Fig. 4b). In this case, because the drug is dispersed in the matrix, diffusion occurred through water filled pores. The release rate showed a positive deviation from linearity (Fig. 4c). From the plot of the drug remaining in the matrix at $24 \mathrm{~h}$ against drug loading, an inflexion point could be determined around 30\% drug loading (Fig. 4d). This result showed that other parameters (like porosity) change with drug loading but not in a proportional fashion. The release mechanism could be explained as follows: at low drug loadings $(<30 \%$ drug loading), pores randomly situated in the matrix are not interconnected and most of the drug is entrapped in the matrix with no diffusion and release possible. When drug loading increases above a critical value ( $>30 \%$ drug loading) pores start to interconnect to form a wet porous network and drug release occurs via diffusion through water filled pores. This critical drug loading is known as the percolation threshold ${ }^{[4]}$. Therefore, only the drug particles accessible to the outside medium through the porous network will contribute to diffusion in the matrix. In other words, isolated drug particles cannot contribute to the transport. When a drug cannot access the matrix surface through the wet pore network, it will not be released. As a result the drug release is slower than predicted, considering aqueous diffusion.

\section{Effect of additives}

\section{Solid solution}

In order to achieve complete drug release from matrix cast films, HPC JF and PVP K90 were used as hydrophilic additives. Increasing HPC JF and PVP K90 content, increased release in similar trend (Fig. 5a and b). Additive content of $7 \%$ and $14 \%$ showed almost no effect of ibuprofen release rate, probably due to similar drug diffusion through the matrices (drug dissolved in the polymers). At $21 \%$ and $28 \%$ of additive, the drug release rate significantly increased (Fig. 5c) due to matrix erosion during drug release studies. This erosion, caused by the additive leaching, shortened the path length for the drug diffusion, increased surface area and the drug release increased. ertação do fármaco pode ser descrita pela raiz quadrada do tempo, o que indica um mecanismo de difusão (Fig. 4b). Neste caso, como o fármaco está disperso, a difusão ocorre através de poros/canais. A velocidade de libertação revelou um desvio positivo à linearidade (Fig. $4 c)$. Através do gráfico de fármaco retido na matriz às $24 \mathrm{~h}$ em função da dosagem, um ponto de inflexão pode ser determinado em torno de $30 \%$ de fármaco (Fig. 4d). Este resultado mostra que outros parâmetros (como a porosidade) também mudam com a dosagem, mas não de uma forma proporcional. O mecanismo de libertação do fármaco pode ser explicado como se segue: a baixas concentrações de fármaco $(<30 \%)$, os poros situados aleatoriamente na matriz não estão interligados e a maior parte do fármaco é aprisionada na matriz, não havendo difusão e libertação possível. Quando a concentração de fármaco aumenta acima de um valor crítico ( $30 \%)$, os poros começam a interligar-se numa rede porosa e a libertação de fármaco ocorre através da difusão por poros/canais embebidos pelo meio de dissolução. Esta dosagem crítica de fármaco é conhecida como o limiar de percolação ${ }^{[4]}$. Como tal, apenas as partículas de fármaco acessíveis ao meio exterior através da rede porosa irão contribuir para a difusão na matriz. Isto é, fármaco isolado não contribui para o transporte. Quando um fármaco não pode aceder à superfície da matriz, através da rede de poros, não será libertado. Por consequência, a libertação é mais lenta do que seria previsível a partir da apreciação simples de difusão aquosa.

\section{Efeito de aditivos}

\section{Solução sólida}

No sentido de atingir libertação completa do fármaco a partir da matriz, HPC JF e PVP K90 foram usados como aditivos hidrofílicos. Com o incremento da concentração de HPC JF e PVP K90, aumentou a libertação de fármaco numa tendência semelhante para os 2 aditivos (Fig. 5a e b). Um conteúdo de aditivo de 7\% e $14 \%$ alterou muito pouco a velocidade de libertação do fármaco, provavelmente devido ao facto da difusão ser muito semelhante nas 2 matrizes (fármaco dissolvido no polímero). Concentrações de $21 \%$ e $28 \%$ aumentaram significativamente a velocidade de libertação (Fig. $5 c$ ), devido à erosão da matriz durante o ensaio. Esta erosão, provocada pela dissolução dos aditivos, encurtou o caminho de difusão para o fármaco, aumentou da área de superfície e a velocidade de libertação também aumentou. 

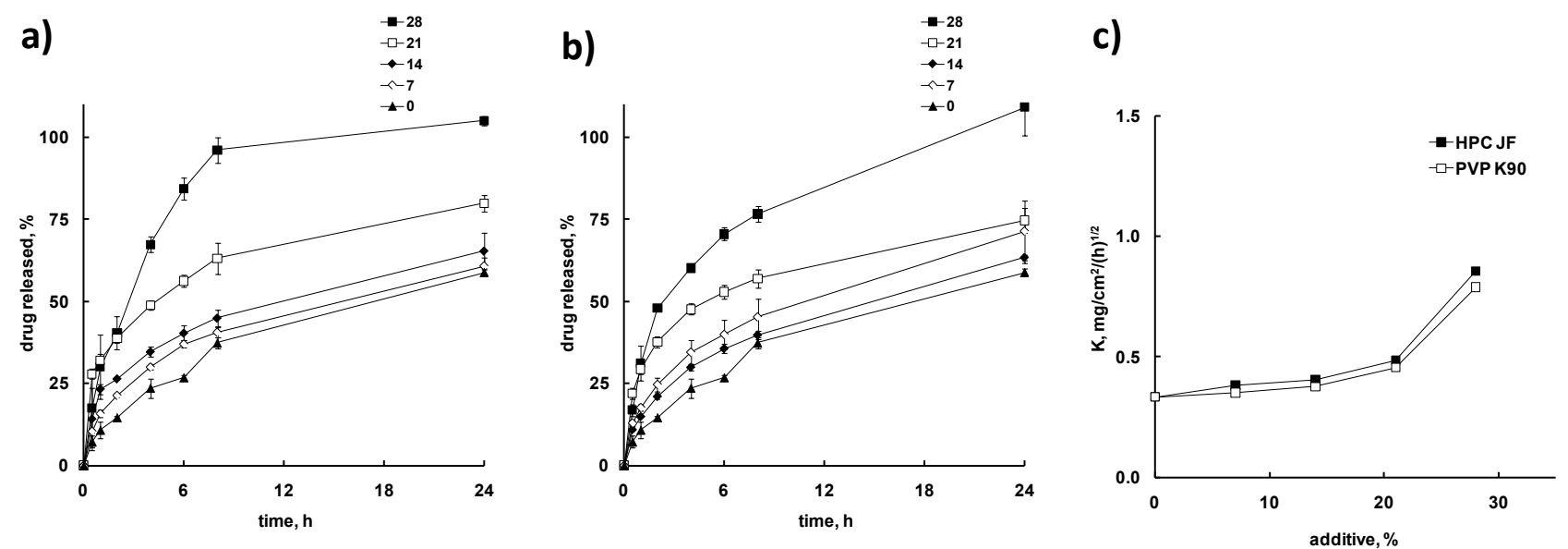

Figure 5 - Effect of additive content (w/w) on ibuprofen release from ethylcellulose matrix cast films (30\% drug loading). a) HPC JF, b) PVP K90 and c) release rate

Figura 5 - Efeito do conteúdo de aditivo $(\mathrm{m} / \mathrm{m})$ na libertação de ibuprofeno dos filmes matriciais de etilcelulose (30\% de dosagem). a) HPC JF, b) PVP K90 e c) velocidade de libertação

\section{$\underline{\text { Solid dispersion }}$}

Metoprolol tartrate release was increased by increasing the HPC JF, PVP K90 and mannitol content in the matrix (Fig. 6a-c). However, the increase in drug release was just in the initial phase. Afterwards, a plateau was reached with all matrices. The increment in the initial release was due to an increase in internal porosity, resulting in faster drug diffusivity through water filled pores and/or channels. The plateau could be explained by entrapment of the drug in the matrix. Consequently, below or at the drug percolation threshold, any optimization of drug release profiles is critical.

\section{Dispersão sólida}

A libertação de tartarato de metoprolol foi potenciada pelo aumento do conteúdo de HPC JF, PVP K90 e manitol (Fig. 6a-c). No entanto, o aumento na libertação de fármaco foi apenas na fase inicial. Posteriormente, foi atingido um plateau para todas as matrizes. O incremento da libertação inicial foi devido a um aumento da porosidade interna, potenciando, por sua vez, a difusão do fármaco através de poros/canais embebidos por meio de dissolução. O plateau pode ser explicado pelo aprisionamento do fármaco na matriz. Por conseguinte, abaixo ou no limiar de percolação, qualquer optimização do perfil de libertação é crítica.
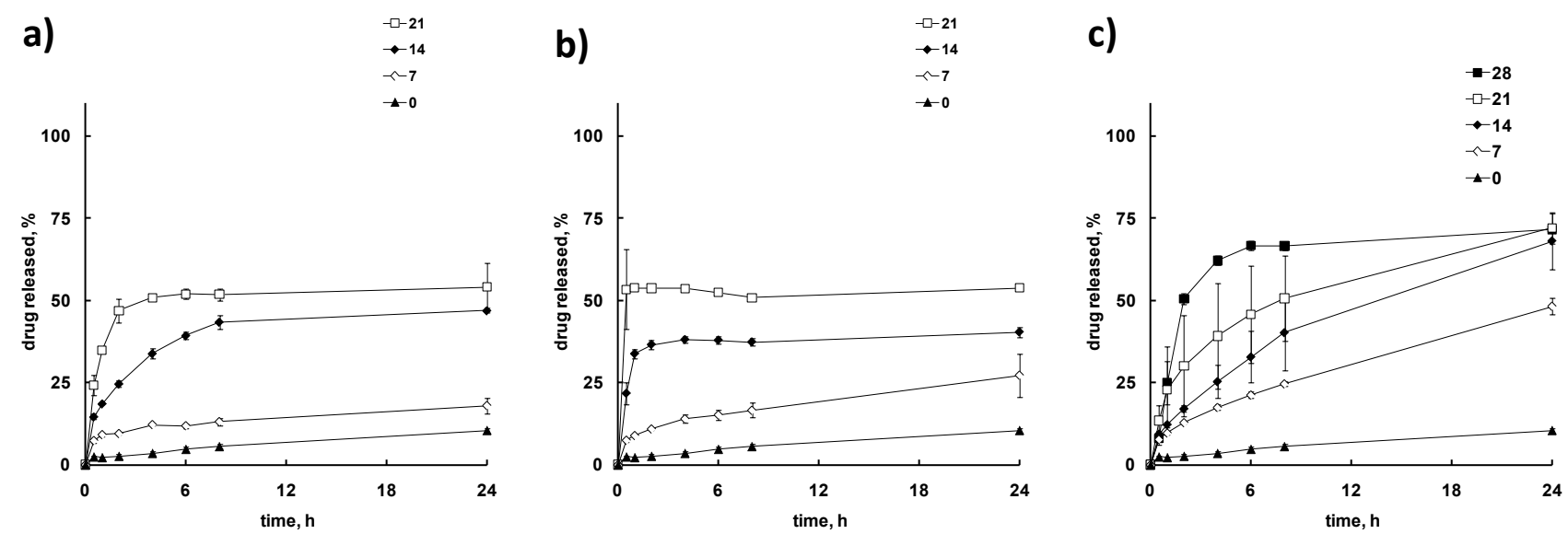

Figure 6 - Effect of additive content (w/w) on metorpolol tartrate release from ethylcellulose matrix cast films (30\% drug loading). a) HPC JF, b) PVP K90 and c) manitol

Figura 6 - Efeito do conteúdo de aditivo $(\mathrm{m} / \mathrm{m})$ na libertação de tartarato de metoprolol dos filmes matriciais de etilcelulose (30\% de dosagem de fármaco). a) HPC JF, b) PVP K90 e c) manitol 
a)

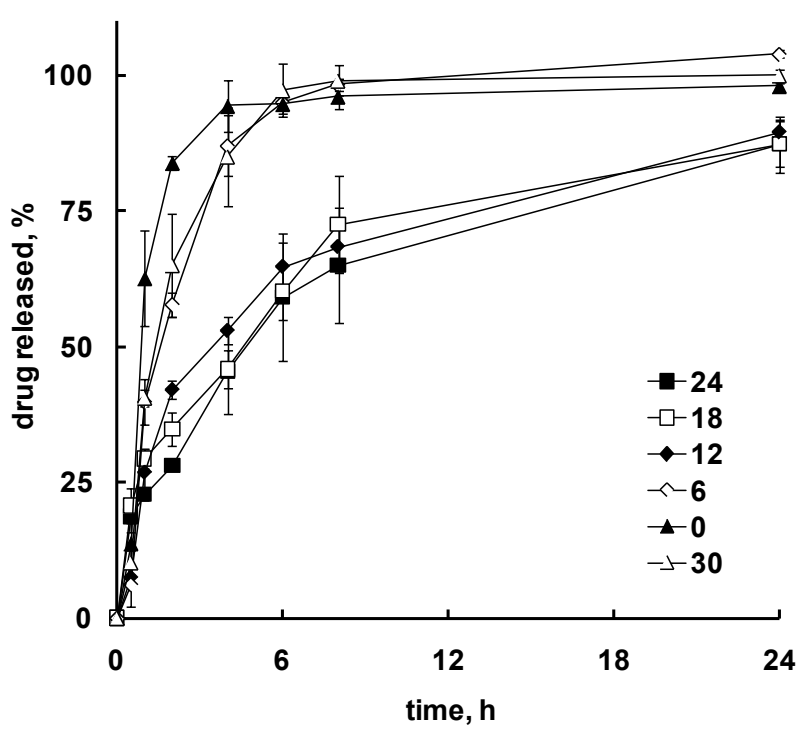

D)

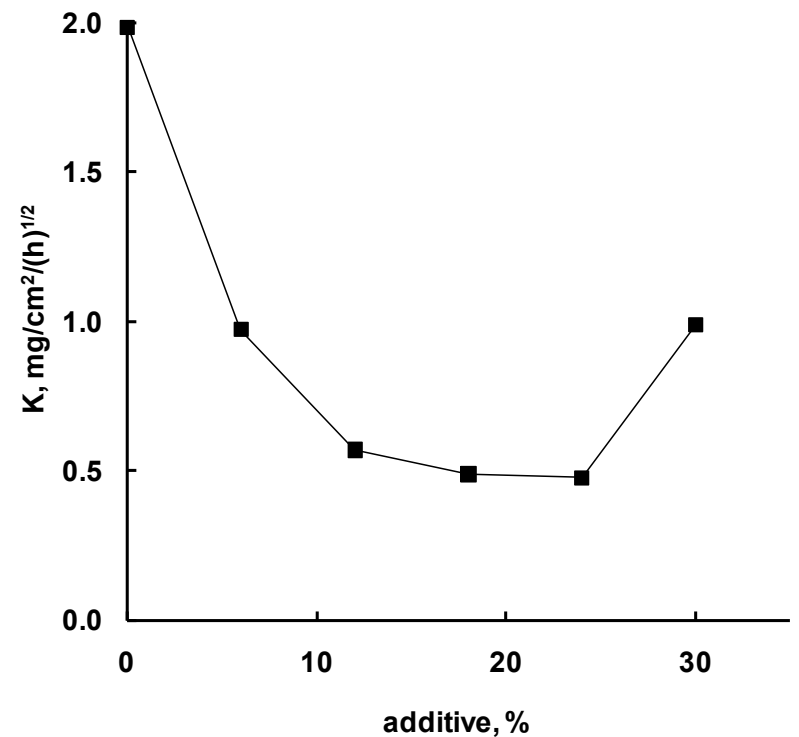

Figure 7 - Effect of PEG 1500 content (w/w) on: a) diclofenac Na release and b) diclofenac Na release rate from ethylcellulose matrix cast films (40\% drug loading).

Figura 7 - Efeito do conteúdo de PEG 1500 (m/m) na: a) libertação de diclofenac $\mathrm{Na}$ e b) constante de libertação de diclofenac $\mathrm{Na}$ dos filmes matriciais de etilcelulose ( $40 \%$ de dosagem de fármaco)

Polyethylene glycol (PEG) is widely used as an additive to increase drug release profiles. Due to its hydrophilicity, it leaches from the films, creating porosity and enhancing drug release profiles. In a few cases PEG was reported to decrease drug release due its affinity to the drug and phase separation with the polymer ${ }^{[5,6]}$. Therefore, this study aimed to decrease diclofenac $\mathrm{Na}$ release from ethylcellulose matrix systems by adding PEG 1500 to the matrix. Diclofenac Na release from ethylcellulose films was very fast, due to the fast dissolution of drug crystals on the matrix surface (Fig. $7 \mathrm{a}, \mathrm{b}$ and $8 \mathrm{a}$ ). The addition of $6 \mathrm{cg} / \mathrm{g}$ of PEG slightly decreased diclofenac Na release; whereas the addition of between $12 \mathrm{cg} / \mathrm{g}$ and $24 \mathrm{cg} / \mathrm{g}$ strongly decreased drug release (Fig. 7a and b). On the contrary, $30 \mathrm{cg} / \mathrm{g}$ PEG increased the drug release rate again due to an incomplete and discontinuous matrix (Fig. 7a and 8d) which contributed to fast drug release. The decreased diclofenac Na release when PEG was added to the matrices was attributed to a better drug entrapment into the ethylcellulose matrices (Fig. 8a-c). Drug solubility, determined by the occurrence of crystals in dried films was $\sim 10 \mathrm{cg} / \mathrm{g}$ in ethylcellulose film and $\sim 60 \mathrm{cg} / \mathrm{g}$ in PEG. PEG itself had a low solubility in ethylcellulose films (around 10\%). When PEG was added to ethylcellulose: diclofenac Na films, very small crystals well distributed over the matrix were seen (Fig. 8 b-d). To
O polietilenoglicol (PEG) é amplamente usado como um excipiente para aumentar a velocidade de libertação do fármaco. Devido à sua hidrofilicidade, é libertado das matrizes criando poros/canais, aumentando assim a libertação dos fármacos. Em alguns casos, o PEG tem sido utilizado para diminuir libertação, devido à sua afinidade com o fármaco, bem como à sua separação da fase do polímero ${ }^{[5,6]}$. Como tal, foi um objectivo do presente estudo diminuir a libertação de diclofenac de $\mathrm{Na}$ das matrizes de etilcelulose, pela adição de PEG 1500. A libertação de diclofenac $\mathrm{Na}$ a partir da matriz de etilcelulose foi muito rápida, devido à rápida dissolução de cristais de fármaco na superfície (Fig. 7a, b e 8a). A adição de $6 \mathrm{cg} / \mathrm{g}$ de PEG diminuiu ligeiramente a libertação de fármaco; enquanto a adição entre $12 \mathrm{cg} / \mathrm{g}$ e $24 \mathrm{cg} / \mathrm{g}$ diminuiu fortemente a libertação de diclofenac $\mathrm{Na}$ (Fig. 7a e b). De forma oposta, $30 \mathrm{cg} / \mathrm{g}$ PEG aumentou novamente a velocidade de libertação, devido à matriz incompleta e descontínua (Fig. 7a e 8d), que contribuiu para a rápida libertação do fármaco. A redução da libertação de diclofenac Na por adição de PEG foi atribuída a um melhor aprisionamento do fármaco nas matrizes de etilcelulose (Fig. 8a-c). A solubilidade da diclofenac Na, determinada pela ocorrência de cristais, foi aproximadamente de $10 \mathrm{cg} / \mathrm{g}$ na matriz de etilcelulose e de $60 \mathrm{cg} / \mathrm{g}$ em PEG. A solubilidade de PEG na matriz de etilcelulose foi baixa (cerca de 10\%). Quando o PEG foi adicionado à matriz etilcelulose:diclofenac de $\mathrm{Na}$, foram observados 


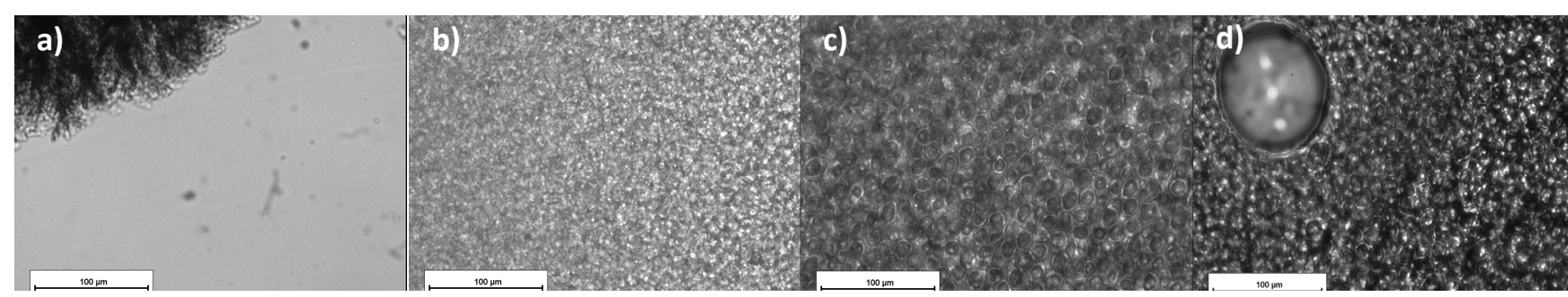

Figure 8 - Polarizing light microscope images from ethylcellulose: diclofenac Na: matrix cast films and different contents of PEG 1500 (w/w) (40\% drug loading). a) 0, b) 12, c) 18 and d) 30.

Figura 8 - Imagens de microscopia de luz polarizada de filmes matriciais de diclofenac Na: etilcelulose com diferentes conteúdos de PEG 1500 (m/m), (40\% dosagem de fármaco). a) 0, b) 12, c) 18 e d) 30 .

determine whether crystallinity was due to diclofenac $\mathrm{Na}$ or PEG, DSC was performed. Since diclofenac Na decomposes immediately after the melting point, it was not possible to interpret the results (data not shown). However, DSC results showed that PEG displayed an amorphous phase after incorporation in ethylcellulose: diclofenac Na matrix (Fig. 9). Therefore, crystals observed in the ternary matrix were due to the drug. These crystals could be dispersed in the matrix or had crystallized out from the PEG microdomains, which acted as drug solubilizer. In the initial phase of drug release, diclofenac $\mathrm{Na}$ was preferentially distributed in PEG pequenos cristais bem distribuídos ao longo da matriz (Fig. 8 b e d). Para determinar se a cristalinidade era devido ao diclofenac de $\mathrm{Na}$ ou PEG, calorimetria diferencial de varrimento (CDV) foi realizada. Uma vez que o diclofenac de Na se decompõe imediatamente após o ponto de fusão, a interpretação dos resultados não foi possível (dados não mostrados). No entanto, os resultados de CDV mostraram que o PEG exibe uma fase amorfa após incorporação na matriz etilcelulose: diclofenac de Na matriz (Fig. 9). Portanto, os cristais observados na matriz ternária são relativos ao fármaco. E estes cristais podem estar dispersos na matriz ou cristalizarem a partir dos domínios de PEG (que

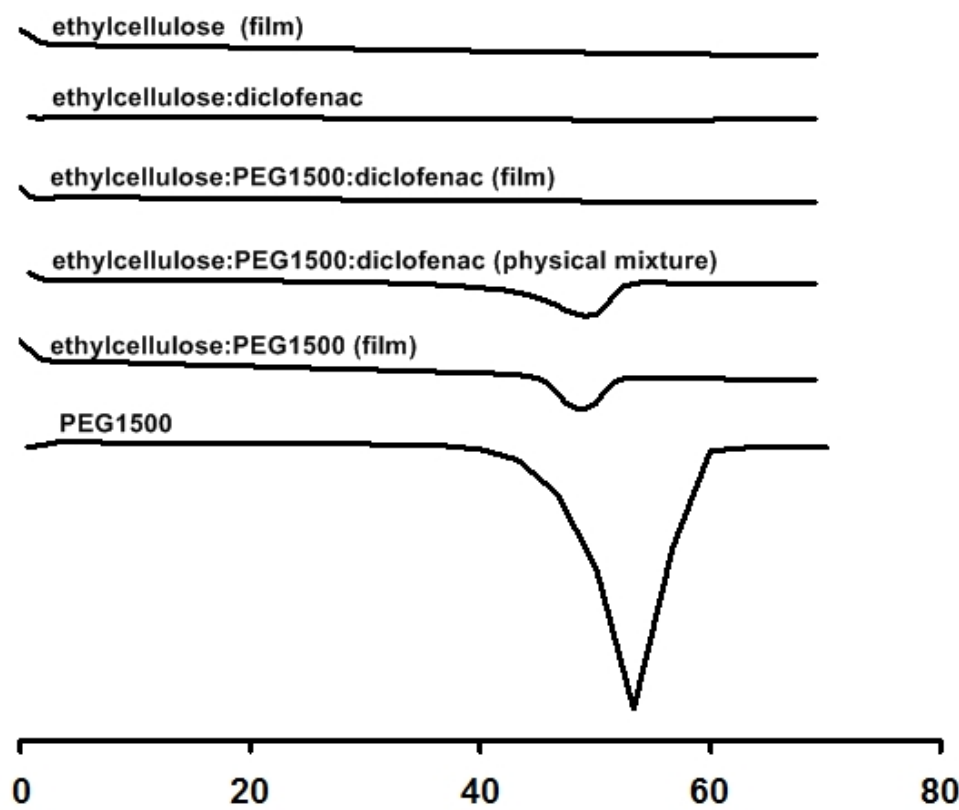

Figure 9 - DSC thermograms (first cycle) of PEG 1500, ethylcellulose film, ethylcellulose: PEG 1500 70:30, ethylcellulose: PEG 1500: diclofenac Na 42:18:40 films and corresponding physical mixtures.

Figura 9 - Termogramas de calorimetria diferencial de varrimento (primeiro ciclo) de PEG 1500, filme de etilcelulose, etilcelulose: PEG 1500 70:30, etilcelulose: PEG 1500: diclofenac Na 42:18:40 filme e as correspondentes misturas físicas. 
domains (ring structures), with better entrapment in the matrix. This accounted for the slower dissolution/ diffusion of diclofenac $\mathrm{Na}$ which resulted in a slower initial drug release. However, complete release was achieved due to PEG leaching, which created porosity and facilitated drug diffusion.

a)

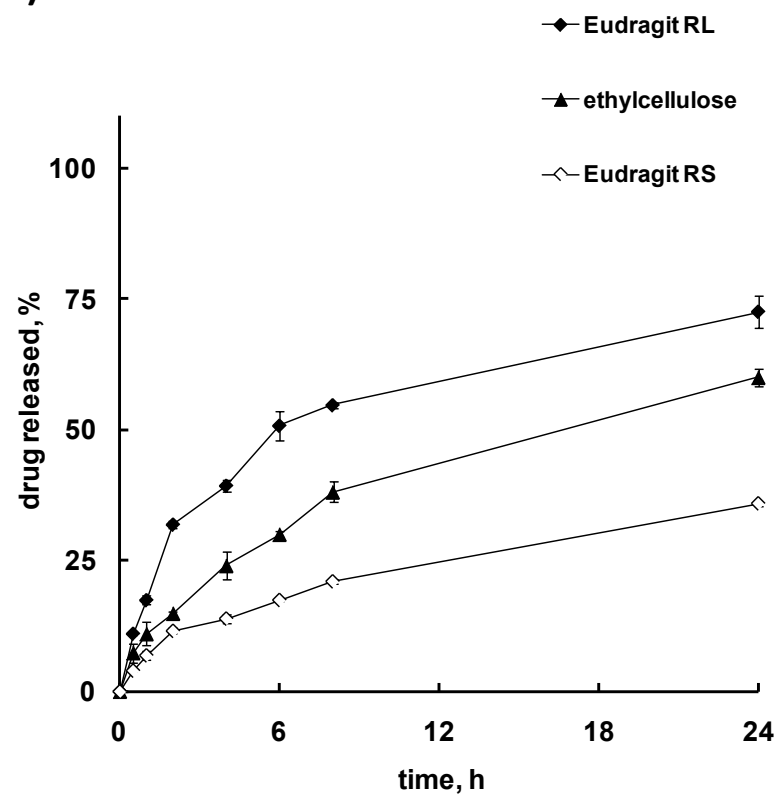

actua como agente solubilizante para o fármaco). Na fase inicial de libertação do fármaco, o diclofenac de $\mathrm{Na}$ estava preferencialmente distribuído nos domínios de PEG (estruturas de anel), com um melhor aprisionamento na matriz, e isto fez-se representar por uma dissolução/difusão mais lenta de diclofenac de $\mathrm{Na}$, resultando numa libertação mais prolongada. No entanto, a libertação completa foi alcançada devido à libertação de PEG (erosão da matriz), que criou porosidade e facilitou a difusão do fármaco.

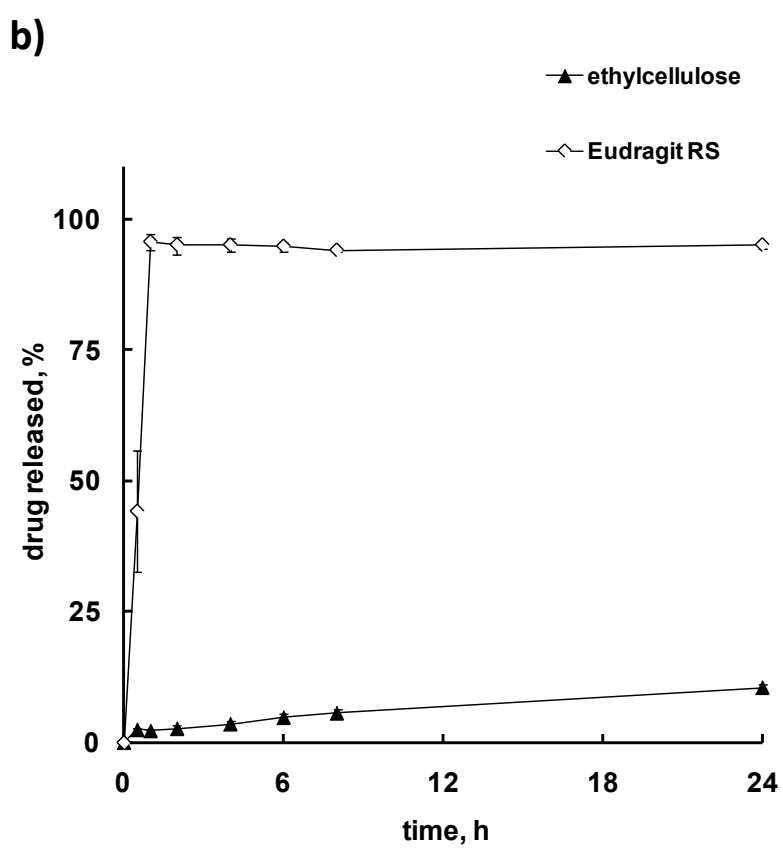

Figure 10 - Effect of polymer type on release of: a) ibuprofen and b) metoprolol tartrate from matrix cast films (30\% drug loading).

Figura 10 - Efeito do tipo de polímero na libertação de: a) ibuprofen e b) tartarato de metoprolol de filmes matriciais (30\% dosagem).

\section{Effect of polymer type}

Ibuprofen release occurred in the following order: Eudragit RL $>$ ethylcellulose $>$ Eudragit RS (Fig. 10a). The drug was dissolved (clear films) in all matrix films and release mechanism occurred by diffusion through the polymer. The drug partition into the polymer was calculated to be: $(73,4 \pm 2,6) \mathrm{cg} / \mathrm{g}$ (Eudragit RL) $>(39,7$ $\pm 2,0) \mathrm{cg} / \mathrm{g}$ (ethylcellulose) $>(3,8 \pm 0,0) \mathrm{cg} / \mathrm{g}$ (Eudragit $\mathrm{RS})$. These values can be interpreted in terms of drug solubility in the polymer. In addition, matrix permeability is a function of drug diffusivity in the matrix and drug partition into the polymer. Thus, drug release order is related to ibuprofen solubility in the polymers and matrix permeability. Metoprolol tartrate release from ethylcellulose was around $10 \%$ in $24 \mathrm{~h}$, in contrast to immediate release from Eudragit RS (Fig. 10b). The drug was dispersed and entrapped in the ethylcellulose

\section{Efeito do tipo de polímero}

A libertação de ibuprofeno foi na seguinte ordem: Eudragit RL $>$ etilcelulose $>$ Eudragit RS (Fig. 10a). O fármaco estava dissolvido em todas as matrizes (filmes transparentes) e o mecanismo de libertação ocorreu por difusão pura através do polímero. A partição de fármaco para o polímero foi calculada como sendo: $(73,4$ $\pm 2,6) \mathrm{cg} / \mathrm{g}$ (Eudragit RL)> $(39,7 \pm 2,0) \mathrm{cg} / \mathrm{g}$ (etilcelulose) $>(3,8 \pm 0,0) \mathrm{cg} / \mathrm{g}$ (Eudragit RS). Estes valores podem ser interpretados em termos de solubilidade do fármaco no polímero. Além disso, a permeabilidade da matriz é uma função da difusão do fármaco na matriz e da partição de droga no polímero. Assim, a libertação de ibuprofeno está relacionada com a solubilidade do fármaco no polímero e permeabilidade da matriz.

A libertação de tartarato de metoprolol da matriz de EC foi de cerca de $10 \%$ em 24 horas, em contraste com a 
matrix and dissolved in the Eudragit RS matrix. Moreover, metoprolol tartrate is able to form an amorphous mixture with Eudragits ${ }^{[1]}$, and is probably the reason for the extremely fast drug release.

\section{Conclusion}

In conclusion, key parameters affecting drug release from solid solutions and solid dispersions were identified. Independent of whether the drug is dissolved or dispersed in the matrix, the drug release profiles from matrix systems were characterized by an initial fast release followed by decreased release rate. In addition, PEG proved to be an efficient excipient to decrease the release rate from diclofenac Na: EC matrix.

\section{Conflict of interests}

The author declares that there are no financial and personal relationships that could be viewed as presenting a potential conflict of interests. libertação imediata de Eudragit RS (Fig. 10b). O fármaco estava disperso e retido na matriz de EC e dissolvido na matriz de Eudragit RS. Além disso, o tartarato de metoprolol é capaz de formar uma mistura amorfa com Eudragits ${ }^{[1]}$ sendo provavelmente a razão para a libertação extremamente rápida do fármaco.

\section{Conclusão}

Foram identificados os principais parâmetros que afectam a libertação do fármaco a partir de soluções sólidas e dispersões sólidas. Independentemente se o fármaco está dissolvido ou disperso na matriz, os perfis de libertação de fármaco a partir de sistemas matriciais são caracterizados por uma libertação inicial rápida seguida por uma diminuição da taxa de libertação. Mais importante, o PEG 1500 provou ser um excipiente eficaz para diminuir a velocidade de libertação de diclofenac $\mathrm{Na}$ a partir da matriz de etilcelulose

\section{Conflito de Interesses}

O autor declara não existir qualquer relação pessoal ou financeira que possa ser entendida como representando um potencial conflito de interesses. 


\section{References / Referências}

[1] Glaessl B, Siepmann F, Tucker I, Siepmann J, Rades T. Characterisation of quaternary polymethacrylate films containing tartaric acid, metoprolol free base or metoprolol tartrate. European Journal of Pharmaceutics and Biopharmaceutics. 2009;73(3):366-72.

[2] Jenquin MR, McGinity JW. Characterization of acrylic resin matrix films and mechanisms of drug-polymer interactions. International Journal of Pharmaceutics. 1994;101(1-2):23-34.

[3] Huang X, Brazel CS. On the importance and mechanisms of burst release in matrixcontrolled drug delivery systems. Journal of Controlled Release. 2001;73(2-3):121-36.
[4] Leuenberger H, Bonny JD, Kolb M. Percolation effects in matrix-type controlled drug release systems. International Journal of Pharmaceutics. 1995;115(2):217-24.

[5] Kang E, Robinson J, Park K, Cheng J-X. Paclitaxel distribution in poly(ethylene glycol)/ poly(lactide-co-glycolic acid) blends and its release visualized by coherent anti-Stokes Raman scattering microscopy. Journal of Controlled Release. 2007;122(3):261-8.
[6] Mu L, Teo M-M, Ning H-Z, Tan C-S, Feng S-S. Novel powder formulations for controlled delivery of poorly soluble anticancer drug: Application and investigation of TPGS and PEG in spray-dried particulate system. Journal of Controlled Release. 2005;103(3):565-75. 